\title{
¿S Research Square

\section{Bixin Attenuates Experimental Autoimmune Encephalomyelitis by Suppressing TXNIP/NLRP3 inflammasome Activity and Activating Nrf2 Signaling}

Ye Yu

Chengdu Medical College

Dong-ming Wu

Chengdu Medical College

Jing Li

Chengdu Medical College

Shi-hua Deng

Chengdu Medical College

Teng Liu

Chengdu Medical College

Ting Zhang

Chengdu Medical College

Miao $\mathrm{He}$

Chengdu Medical College

Yang-yang Zhao

Chengdu Medical College

Ying Xu ( $\nabla$ yingxu825@126.com )

Chengdu Medical College https://orcid.org/0000-0001-9570-8666

\section{Research}

Keywords: Bixin, Neuroinflammation, Demyelination, Experimental autoimmune encephalomyelitis, TXNIP, NLRP3, Nuclear factor erythroid 2-related factor 2, Reactive oxygen species

Posted Date: April 28th, 2020

DOl: https://doi.org/10.21203/rs.3.rs-24795/v1

License: (c) (1) This work is licensed under a Creative Commons Attribution 4.0 International License.

Read Full License 


\section{Abstract}

Background Multiple sclerosis (MS), an autoimmune and degenerative disease, is characterized by demyelination and chronic neuroinflammation.Bixin is a carotenoid isolated from the seeds of Bixa orellana that exhibits various potent pharmacological activities, including antioxidant, anti-inflammation, and anti-tumor. However, the effects of bixin on MS remain elusive.

Methods To evaluate the effects and underlying molecular mechanisms of bixin on MS, experimental autoimmune encephalomyelitis (EAE) was established in C57BL/ 6 mice, which were treated with intragastric administration of bixin solutions. To evaluate the molecular mechanisms of bixin, quantitative reverse-transcription PCR, western blot, immunohistochemistry, and enzyme-linked immunosorbent assay analyses were performed.

Results We found that bixin significantly improved the symptoms in EAE mice, and suppressed microglia aggregation and TXNIP/NLRP3 inflammasome activity by scavenging excessive reactive oxygen species (ROS). Furthermore, bixin activated nuclear factor erythroid 2-related factor 2 (Nrf2) and its downstream genes in EAE mice, and these effects were suppressed upon inhibiting Nrf2 expression with the Nrf2 inhibitor ML385.

Conclusions Bixin prevented neuroinflammation and demyelination in EAE mice mainly by scavenging ROS through activation of the Nrf2 signaling pathway. Taken together, our results indicate that bixin is a promising therapeutic candidate to treat MS.

\section{Background}

Multiple sclerosis (MS) is a chronic autoimmune and degenerative disease of the central nervous system (CNS), and the most frequent cause of neurological disability in young adults [1]. The pathological changes in patients with MS are multifaceted, including multiple demyelinating plaques, and are accompanied by glial activation and axonal damage [2-6]. Although many studies have proposed possible molecular mechanisms and therapeutic strategies for MS, its pathology is largely unknown, involving apoptosis, oxidative stress, and inflammation, and effective treatments remain elusive $[7,8]$.

A recent study has proven that the NLRP3 inflammasome plays a pivotal role in the pathogenesis of neuroinflammation and demyelination in EAE [5]. The thioredoxin-interacting protein (TXNIP)-NLRP3 inflammasome is a macromolecular polyprotein complex, which is composed of TXNIP, NLRP3, the junction protein ASC, and the effector protein Caspase-1. Excessive reactive oxygen species (ROS) accumulation induced by high glucose increases TXNIP expression. Accumulated TXNIP, in turn, activates the NLRP3 inflammasome, consequently inducing the secretion of IL-1 $\beta$ and IL-18 as a part of the inflammatory response [9-12]. Furthermore, activated NLRP3 inflammasome induces the expression of gasdermin D (GSDMD), which leads to the release of numerous types of inflammatory cytokines, exacerbating the detrimental inflammatory response [13]. Therefore, blocking TXNIP-NLRP3 activity is expected to alleviate neuroinflammation and demyelination. 
Bixin is a natural chemo-preventive carotenoid isolated from the seeds of Bixa orellana and can cross the blood-brain barrier [14-16]; it is a lineal apocarotenoid of 25 carbon atoms with 9 double bonds. It has been demonstrated to exhibit various potent pharmacological activities, including antioxidant, antiinflammation, and anti-tumor $[17,18]$. Previous studies have demonstrated that bixin can ameliorate high-fat-diet-induced cardiac injury and PM2.5 particle-induced lung injury through suppressing inflammation and oxidative stress $[19,20]$. The present study evaluated the effects of bixin on neuroinflammation and demyelination in a mouse model of autoimmune encephalomyelitis, with the aim of assessing whether bixin could be an effective therapeutic compound for MS.

\section{Methods}

\section{Antibodies}

The primary antibodies used for western blot assay and immunohistochemical analysis were as follows: anti-GAPDH (Proteintech, Wuhan, China); anti-Catalase (CAT), anti-SOD2 (Cell Signaling Technology, Danvers, MA, USA); anti-3-Nitrotyrosine (3-NT, Millipore); anti-NAD (P)H dehydrogenase quinone 1 (NQ0-1), anti-Nrf2, anti-TXNIP, anti-NLRP3, anti-Caspase-1, anti-IL-18, anti-IL-1 $\beta$, and anti-ASC (Abcam, Cambridge, UK). Horseradish peroxidase (HRP)-conjugated secondary antibodies used for western blot analysis were purchased from Proteintech (Wuhan, China). The SPlink Detection Kits used for immunohistochemical analysis were purchased from ZSGB-BIO Technology (Beijing, China).

\section{Establishment Of The Eae Model}

Seven-week-old female C57BL/6 mice were purchased from Chengdu Dashuo Experimental Animal Company (Chengdu, China). All mice were housed in a specific pathogen-free facility with a 12-h light/dark cycle and provided with regular food and water for 1 week before any experiments.

The EAE model was established as previously described [21]. Myelin oligodendrocyte glycoprotein ${ }_{35-55}$ $\left(\mathrm{MOG}_{35}-55\right)$ peptide (Hook Labs, USA) $(200 \mu \mathrm{g})$ was dissolved in 100- $\mu \mathrm{L}$ phosphate-buffered saline (PBS) and emulsified with $100 \mu \mathrm{L}$ of complete Freund's adjuvant (CFA; Chondrex, USA) supplemented with $400 \mu \mathrm{g}$ Mycobacterium tuberculosis H37Ra (Difco, BD Biosciences, USA). Then, the above emulsions were subcutaneously injected (Day 1). Pertussis toxin (PTX; List Biological Labs, Campbell, CA) (300 ng) was intraperitoneally administered on the first and third days post-immunization.

\section{Bixin Treatment}

Animals were randomly divided into the following 5 groups: healthy control (PBS, $n=5), E A E(n=5), E A E$ + bixin (50 mg/kg, once daily; $n=5)$, $E A E+$ bixin (100 mg/kg, once daily; $n=5)$, and $E A E+$ bixin (200 mg/kg, once daily; $\mathrm{n}=5$ ). Bixin was dissolved in dimethyl sulfoxide (DMSO) at $2 \mathrm{~g} / \mathrm{mL}$ and then diluted with PBS. Mice in EAE + bixin group were treated with intragastric administration of bixin solutions 
at a dose of 50,100 , or $200 \mathrm{mg} / \mathrm{kg}$ daily from the $3 \mathrm{rd}$ day to the 15 th day. On the 15 th day, the mice from each group were euthanized; the brains and spinal cords of the mice were collected.

\section{Nrf2 Inhibitor Treatment}

The Nrf2 inhibitor ML385 (MCE, Shanghai, China) was dissolved in DMSO at $300 \mathrm{mg} / \mathrm{mL}$ and diluted with PBS [22]. Mice were randomly assigned to the following 8 groups: healthy control (PBS, $n=5), E A E$ $(n=5), E A E+$ bixin $(100 \mathrm{mg} / \mathrm{kg}$, once daily; $n=5)$, bixin $(100 \mathrm{mg} / \mathrm{kg}$, once daily; $n=5), \operatorname{ML385}(n=5)$; ML385 + EAE ( $(n=5), M L 385+E A E+$ bixin $(100 \mathrm{mg} / \mathrm{kg}$, once daily; $\mathrm{n}=5)$, and ML385 + bixin $(100 \mathrm{mg} / \mathrm{kg}$, once daily; $\mathrm{n}=5)$. ML385 (30 mg/kg) pre-treatment was intraperitoneally administered $1 \mathrm{~h}$ before intragastric administration of bixin.

\section{Bodyweight And Behavioral Assessments}

Clinical behavior scores of each group were evaluated as per the following criteria: 0 , without symptoms; 1, lost tail tension; 2 , flaccid hind limb; 3 , moderate hind limb paralysis; 4 , paralysis of both hind limbs and forelimbs, or accompanied with urinary and fecal disorders; and 5, near-death state [2]. The body weight was recorded daily.

\section{Hematoxylin and Eosin (HE) and luxury fast blue (LFB) Staining}

Mice were euthanized under anesthesia, and the brain and spinal cord were fixed with $4 \%$ paraformaldehyde (in PBS) for $24 \mathrm{~h}$ at room temperature, dehydrated with an ethanol gradient and cleared with xylene, subsequently embedded in paraffin, and then cut into 5 - $\mu \mathrm{m}$ sections.

To evaluate the degree of inflammatory cell infiltration, brain sections were stained using an HE staining kit (Beyotime Biotechnology, China, Shanghai). The sections were dewaxed and dehydrated, subsequently washed with PBS, and then stained with hematoxylin and eosin for 2 min, respectively.

The spinal cords were stained with LFB staining solution (Solarbio, Beijing, China), to evaluate changes in demyelination. The sections were stained with modified page staining solution and page peach red dye solution, respectively, after being dewaxed and dehydrated with an ethanol gradient.

The inflammatory infiltration and demyelination scores were evaluated as previously reported [23]. Images of brain or spinal cord sections were randomly captured at $20 \times$ magnification (XI 71 Olympus, Tokyo, Japan).

\section{Immunohistochemical Analysis (ihc)}


IHC was performed using an SPlink Detection Kit (ZSGB-BIO Technology, Beijing, China). Tissue sections were dewaxed and dehydrated, and washed with PBS. After washing, the samples were boiled in a citrate buffer ( $\mathrm{pH}$ 6.0) for antigen retrieval, and blocked using $5 \%$ normal goat serum at $37^{\circ} \mathrm{C}$ for $1 \mathrm{~h}$. Subsequently, the sections were incubated at $4{ }^{\circ} \mathrm{C}$ overnight with primary antibodies (1:200). After washing with PBS, the sections were then incubated with the corresponding secondary antibody for $30 \mathrm{~min}$. Finally, diaminobenzidine was used as the chromogen to visualize the immunocomplexes, and then the sections were counterstained with hematoxylin. Images of the random brain sections were captured at $40 \times$ magnification (XI 71 Olympus, Tokyo, Japan).

\section{Quantitation Of Oxidative Stress}

Dihydroethidium (DHE; Molecular Probes, Eugene, OR) staining was used to detect the ROS levels in the brain and spinal cord. The sections were dewaxed and dehydrated with an ethanol gradient, after washing with PBS (pH 7.4), tissue sections were blocked using $5 \%$ BSA for 30 min at $37^{\circ} \mathrm{C}$, and stained with $5 \mu \mathrm{mol} / \mathrm{L} \mathrm{DHE}$ (in PBS) for $30 \mathrm{~min}$ at $37^{\circ} \mathrm{C}$. The ROS levels in EAE mice in the absence or presence of bixin were likewise evaluated by DHE staining. Finally, fluorescence images of brain or spinal cord sections were randomly captured at $40 \times$ magnification (XI 71 Olympus, Tokyo, Japan).

\section{ELISA}

Mice were euthanized under anesthesia, and the brain were collected and placed in PBS (pH 7.4) on ice and homogenized using a homogenizer. The homogenates were centrifuged at $3000 \mathrm{rpg}$ for $20 \mathrm{~min}$, and then the supernatants were collected. A portion of each supernatant was used for quantitating $\mathrm{IL}-1 \beta$ and IL-18 levels by ELISA, and the rest was maintained $-80^{\circ} \mathrm{C}$ for further analysis. A commercial kit was used for the ELISA assays (MIBIO Biotechnology, Shanghai, China).

\section{Measurement Of Superoxide Dismutase (sod) And Catalase (cat) Activities}

The activities of SOD and CAT in brain tissues (The Institute of Biological Engineering of Nanjing Jiancheng, Nanjing, China) were measured according to the manufacturer's instructions.

\section{Quantitative Reverse-transcription Pcr (qrt-pcr)}

Total brain RNA was extracted with a total RNA extraction kit (Solarbio, Beijing, China) according to the manufacturer's instructions. Then, the cDNA was synthesized using an iScript cDNA synthesis kit (BioRad, Hercules, CA, USA). Nrf2, Catalase, NQO-1, TXNIP, NLRP3, ASC, Caspase-1, IL-18, IL-1 $\beta$ and SOD2 mRNA levels were analyzed by qRT-PCR with SYBR Green Supermix (Bio-Rad, Hercules, CA, USA). The 
primers were synthesized by Shanghai Shenggong and listed in Table 1 ( $\beta$-actin was used as an internal control for quantitation). The $2^{-\Delta \Delta C T}$ method was used to calculate relative mRNA levels.

Table 1

Primer sequence information

\begin{tabular}{|lll|}
\hline Genes & Forward primers $\left(\mathbf{5}^{\prime} \rightarrow \mathbf{3}^{\prime}\right)$ & Reverse primers $\left(\mathbf{5}^{\prime} \rightarrow \mathbf{3}^{\prime}\right)$ \\
\hline Actin & GTGCTATGTTGCTCTAGACTTCG & ATGCCACAGGATTCCATACC \\
\hline IL-6 & CTTGGGACTGATGCTGGTGACAAC & AGGTCTGTTGGGAGTGGTATCCTC \\
\hline IL-8 & CATGGGTGAAGGCTACTGTTGGC & GCTTCATTGCCGGTGGAAATTCC \\
\hline IL-10 & TCTACTGAACTTCGGGGTGATCGG & GTGGTTTGTGAGTGTGAGGGTCTG \\
\hline Nrf2 & TAAAGCACAGCCAGCACATTCTCC & TGATGACCAGGACTCACGGGAAC \\
\hline NQ0-1 & GCTGGTTTGAGAGAGTGCTCGTAG & CCCGTGGACACCCTGAAGAGAG \\
\hline Catalase & GGAGGCGGGAACCCAATAGGAG & TCAAAGTGTGCCATCTCGTCAGTG \\
\hline NLRP3 & GAGCTGGACCTCAGTGACAATGC & ACCAATGCGAGATCCTGACAACAC \\
\hline Caspase1 & CATCCTGTCAGGGGCTCACTTTTC & CTATCAGCAGTGGGCATCTGTAGC \\
\hline ASC & GAAGTGGACGGAGTGCTGGATG & CTTGTCTTGGCTGGTGGTCTCTG \\
\hline TXNIP & CCCAGATACCCCAGAAGCTCCTC & TGAGAGTCGTCCACATCGTCCAG \\
\hline IL-1 $\beta$ & CAAGAGCTTCAGGCAGGCAGTATC & AGGTCCACGGGAAAGACACAGG \\
\hline IL-18 & GGCTGCCATGTCAGAAGACTCTTG & AGTGAAGTCGGCCAAAGTTGTCTG \\
\hline SOD2 & AGCCGTGTCTGTGGGAGTCC & AGAGCAGGCAGCAATCTGTAAGC \\
\hline
\end{tabular}

\section{Western Blot Assay}

Brain tissues were lysed in ice-cold RIPA lysis buffer (Beyotime Biotechnology, Shanghai, China). The protein concentration was determined using a BCA reagent kit (Beyotime Biotechnology, Shanghai, China). Total protein $(30 \mu \mathrm{g})$ was separated by $10 \%$ sodium dodecyl sulfate-polyacrylamide gel electrophoresis, and transferred onto PVDF membranes (Millipore, Billerica, MA, USA). The membranes were blocked in tris-buffered saline with $5 \%$ non-fat milk and $0.5 \%$ bovine serum albumin for $1 \mathrm{~h}$ at room temperature and then incubated overnight at $4{ }^{\circ} \mathrm{C}$ with primary antibodies $(1: 1,000)$. After washing, the membranes were incubated with secondary antibodies $(1: 5,000)$ for $1 \mathrm{~h}$ at $37^{\circ} \mathrm{C}$. Blots were visualized with the Chemiluminescent HRP substrate (Millipore) and quantified with the Quantity 5.2 software System (Bio-Rad). 


\section{Statistical Analyses}

All data are expressed as mean \pm SD. Statistical analysis was performed using GraphPad Prism 7.0 software (GraphPad, San Diego, CA, USA) with one-way ANOVA, followed by post-hoc multiple comparisons with the Tukey's test. Statistical significance was considered as $p<0.05$.

\section{Results}

\section{Bixin attenuates the symptoms of EAE mice}

Mice in the EAE mouse model group lost weight, had increased clinical behavioral scores (Fig. 1a, b), and had more inflammatory cells and demyelination than mice in the healthy control group (Fig. 1c, d).

To evaluate the effects of bixin on the symptoms of EAE mice, daily weight changes and clinical behavioral scores were recorded. The results showed that bixin treatment significantly delayed the detrimental effects of EAE on body weight by the 9th day of the treatment (Fig. 1g). The clinical behavioral scores were significantly increased at day 9 in EAE group compared with the control group, and bixin treatment dose-dependently reduced the clinical symptoms in the EAE mice (Fig. $1 \mathrm{~h}$ ). These results indicated that the appropriate dose of bixin to attenuate the symptoms of EAE was $100 \mathrm{mg} / \mathrm{kg}$, which we used in the following experiments.

\section{Bixin reduces the inflammation and demyelination in EAE mice}

Previous studies have indicated that inflammatory response and demyelination are the main features of EAE mice [5]. Our results showed that EAE significantly increased the number of inflammatory cells and inflammation scores in the brain, but bixin treatment noticeably reduced the aggregation of inflammatory cells and inflammation scores (Fig. 2a). Furthermore, LFB staining revealed that bixin treatment noticeably prevented the demyelination of the spinal cord in EAE mice (Fig. 2b).

Given that inflammatory cytokines play key roles in inflammation, we also evaluated the levels of several cytokines by qRT-PCR. The results revealed that the mRNA levels of the pro-inflammatory cytokines TNFa, IL-6, and IL-8 were markedly increased in the brain of EAE mice, while that of the anti-inflammatory cytokine IL-10 was decreased; bixin treatment reduced the mRNA levels of TNF-a, IL-6, and IL-8, and increased the mRNA level of IL-10 (Fig. 2c-f).

Since microglia are the main mediators of neuronal inflammation [24] and the lysosomal protein CD68 is highly expressed on the surface of activated microglia [5], we evaluated CD68 levels by IHC. In line with the above results, although EAE significantly upregulated CD68, bixin treatment remarkably suppressed this phenotype (Fig. 2g). 


\section{Bixin suppresses the activation of the TXNIP/NLRP3 inflammasome in EAE mice}

To clarify whether bixin suppressed the inflammatory response in EAE mice by inhibiting the activation of the TXNIP/NLRP3 inflammasome, we evaluated the levels of the protein components of this complex in the brain by western blotting. The results showed that TXNIP, NLRP3, ASC, Caspase-1, IL-1 $\beta$, and IL-18 levels were increased in EAE mice, and bixin treatment suppressed their upregulation (Fig. 3a). We also evaluated the mRNA levels of these components by qRT-PCR. Similar to the results at the protein level, bixin suppressed the upregulation of the mRNA levels of all these inflammasome components (Fig. $3 \mathrm{~b}-\mathrm{g}$ ). Furthermore, as shown in Fig. 3h, i, tissue IL-1 $\beta$ and IL-18 levels were increased in EAE mice, and bixin treatment reduced their upregulation. These findings were corroborated by IHC; although NLRP3 and TXNIP were highly expressed in the brains of EAE mice, bixin treatment remarkably inhibited their expression (Fig. 3j, k).

\section{Bixin Inhibits Oxidative Stress In Eae Mice}

To elucidate whether the anti-inflammatory effect of bixin occurred through an anti-oxidative effect, we evaluated the ROS levels in the brain and spinal cord by DHE staining. The results showed that the corresponding ROS levels were significantly increased in EAE mice, and bixin treatment alleviated this oxidative stress (Fig. 4a). Additionally, western blotting results showed that the level of the oxidative damage marker 3-NT in EAE mice was reduced by bixin treatment (Fig. 4b). In corroboration, the reduced CAT and SOD activities in EAE mice were suppressed by bixin treatment (Fig. 4c, d).

\section{Bixin Activates The Nrf2 Signaling In Eae Mice}

Since Nrf2 plays a critical role in oxidative stress response [25], we assessed whether bixin attenuated the oxidative stress in EAE mice via activating Nrf2. Toward this end, we evaluated the levels of the Nrf2 signaling components by western blotting. The results showed that although the levels of Nrf2 and its downstream proteins catalase, NQO-1 and SOD2 were reduced in EAE mice, bixin treatment significantly upregulated them (Fig. 5a). The mRNA levels had the same trend as the protein levels (Fig. 5b-e). Furthermore, IHC indicated that Nrf2 expression was significantly reduced in EAE mice, and bixin treatment reversed this phenotype (Fig. 5f).

To confirm the pivotal role of Nrf2 in the bioactivity of bixin, the Nrf2 inhibitor ML385 was intraperitoneally administered $1 \mathrm{~h}$ before intragastric administration of bixin, which significantly decreased the expression of Nrf2 and its downstream genes, and increased the levels of ROS; bixin could not reverse this phenotype in ML385-treated EAE mice (Fig. 5).

\section{Discussion}


MS is a characteristic autoimmune inflammatory demyelinating disease of the CNS that seriously impacts the quality of life of those affected [26]. While numerous drugs are available for MS treatment, their efficacy remains unsatisfactory. Dang $C$ et al. [27] have indicated that upregulating PGC-1a significantly improves the survival ability of neurons in EAE via inhibiting oxidative stress. Additionally, ghrelin decreases neuroinflammation and demyelination by inhibiting the NLRP3 inflammasome activity [5]. In this study, we similarly demonstrated that NLRP3 inflammasome activity and oxidative stress were the key factors in the development of EAE.

Bixin is a natural carotenoid with multiple bioactivities. Previous studies have shown that bixin has antiinflammation, anti-tumor, and anti-oxidative effects [28-32]. Xu Z et al. [19] have found that bixin attenuates cardiac injury by inhibiting inflammation and oxidative stress in a high-fat-diet mouse model. In this study, we found that bixin suppressed inflammatory cell infiltration and the levels of TNF-a, IL-6, and IL-8, and upregulated the content of IL-10. Microglia are resident immune effector cells in the brain, which are easily activated, leading to a chronic neuroinflammatory reaction in the brain, and are closely related to the occurrence and development of neurodegenerative diseases, such as Alzheimer's disease $[29,33]$. In our study, we found that EAE upregulated the expression of CD68, a marker of activated microglia; bixin was found to reduce microglia activation in the brain. These results indicate that bixin improved the symptoms of EAE mice and demyelination by inhibiting inflammatory cell infiltration and the production of inflammatory cytokines.

The TXNIP/NLRP3 inflammasome plays a key role in the pathogenesis of various diseases [30,32, 34, 35]. Chen $\mathrm{W}$ et al. [9] have indicated that minocycline improves diabetic retinopathy by inhibiting the TXNIP/NLRP3 inflammasome pathway, and Vitamin D3 attenuates diabetic retinopathy by inhibiting high-glucose-induced ROS/TXNIP/NLRP3 inflammasome pathway [36]. In the present study, we found that the TXNIP/NLRP3 inflammasome components were upregulated in EAE mice, and bixin remarkably downregulated the expression of TXNIP and NLRP3 at both mRNA and protein levels. These results indicate that bixin reduced neuroinflammation in EAE by suppressing the activation of the TXNIP/ NLRP3 inflammasome.

Recent studies have reported that oxidative stress is one of the main reasons for CNS dysfunction in MS [27]. ROS are the main mediators of oxidative stress and are initiators of the TXNIP/NLRP3 inflammasome [37-39]. In our study, we found that bixin attenuated ROS accumulation and downregulated the oxidative damage marker 3-NT in EAE mice. These results indicate that bixin suppressed the activation of the TXNIP/NLRP3 inflammasome through the attenuation of oxidative stress.

Nrf2, an important redox sensor, binds to antioxidant response elements and counteracts the production of ROS by activating the expression of many antioxidant genes $[40,41]$. Some studies have shown that bixin alleviates photodamage and hair graying by activating Nrf2 signaling [42]. Another study found that bixin promotes tissue repair and improves pulmonary injury induced by particle exposure in an Nrf2dependent manner [20,43]. In our study, the results showed that bixin markedly upregulated Nrf2 and its 
downstream antioxidant target genes in EAE mice. These results indicate that bixin suppressed the production of ROS by activating Nrf2 signaling. Furthermore, we found that the protective role of bixin was dependent on Nrf2 activation, as the Nrf2 inhibitor ML385 almost completely abrogated the effects of bixin on ROS attenuation. Therefore, these findings indicate that bixin prevented neuroinflammation and demyelination in EAE mice mainly by scavenging ROS through activation of the Nrf2 signaling pathway.

\section{Conclusions}

In conclusion, bixin inhibits the TXNIP/NLRP3 inflammasome and activates the Nrf2 signaling pathway in EAE mice. While bixin may be a possible therapeutic strategy for MS, future mechanistic studies of bixin on neurons in vitro are necessary.

\section{Abbreviations}

ANOVA

Analyses of variance; CFA:Complete Freund's adjuvant; CNS:Central nervous system; MS:Multiple sclerosis; EAE:Experimental autoimmune encephalomyelitis; HE:Hematoxylin and Eosin; LFB:Luxury fast blue; PBS:Phosphate-buffered saline; DHE:Dihydroethidium; ROS:Reactive oxygen species; Nrf2:Nuclear factor (erythroid derived 2)-like 2; CAT:Catalase; NQO-1:NAD(P)H dehydrogenase (quinone 1); 3-NT:3Nitrotyrosine; TXNIP:The thioredoxin-interacting protein; NLRP3:NOD-like receptor 3; IL-1 $\beta$ :Interleukin 1 $\beta$; IL-18:Interleukin 18; TNF-a:Tumor necrosis factor-a; IL-6:Interleukin 6; IL-8:Interleukin 8; IL-10:Interleukin 10 .

\section{Declarations}

\section{Ethics approval and consent to participate}

All experimental procedures were approved by Chengdu Medical College Ethics Committee (CDYXY2019036).

\section{Consent for publication}

Not applicable.

\section{Availability of data and materials}

All data generated or analyzed during this study are included in this published article.

\section{Competing interests}

The authors declare that they have no competing interests. 


\section{Funding}

This study was funded by the National Natural Science Foundation of China (81972977 and 81802955$)$, Foundation of Sichuan Science and Technology Agency (2018JY0648 and 2019YJ0589), Foundation of The First Affiliated Hospital of Chengdu Medical College (CYFY2017ZD03 and CYFY2018ZD02), Foundation of Chengdu Medical College (CYCG16-04), Foundation of Collaborative Innovation Center of Sichuan for Elderly Care and Health, Chengdu Medical College (19Z01), and the Foundation of Chengdu Medical College (CYFY2017YB08 and CYFY2019ZD06).

\section{Authors' contributions}

YY and DW researched data; YY and DW wrote, reviewed, and edited the manuscript; JL,SD, TL, TZ, MH, and $Y Z$ provided research material and techniques; $Y X$ directed the project, and wrote, reviewed, and edited the manuscript. All authors read and approved the final manuscript

\section{Acknowledgments}

Not applicable.

\section{References}

1. Williams SK, Fairless R, Maier O, Liermann PC, Pichi K, Fischer R, et al. Anti-TNFR1 targeting in humanized mice ameliorates disease in a model of multiple sclerosis. Sci Rep. 2018;8:13628.

2. De Paula ML, Rodrigues DH, Teixeira HC, Barsante MM, Souza MA, Ferreira AP. Genistein downmodulates pro-inflammatory cytokines and reverses clinical signs of experimental autoimmune encephalomyelitis. Int Immunopharmacol. 2008:8:1291-7.

3. Ascherio A, Munger KL. Environmental risk factors for multiple sclerosis. Part l: the role of infection. Ann Neurol. 2007;61:288-99.

4. O'Gorman C, Lin R, Stankovich J, Broadley SA. Modelling genetic susceptibility to multiple sclerosis with family data. Neuroepidemiology. 2013;40:1-12.

5. Liu F, Li Z, He X, Yu H, Feng J. Ghrelin attenuates neuroinflammation and demyelination in experimental autoimmune encephalomyelitis involving NLRP3 inflammasome signaling pathway and pyroptosis. Front Pharmacol. 2019;10:1320.

6. Correale J, Gaitán MI, Ysrraelit MC, Fiol MP. Progressive multiple sclerosis: from pathogenic mechanisms to treatment. Brain. 2017;140:527-46.

7. Zhang D, Qiao L, Fu T. Paricalcitol improves experimental autoimmune encephalomyelitis (EAE) by suppressing inflammation via NF-KB signaling. Biomed Pharmacother. 2020;125:109528.

8. Pejman S, Kamarehei M, Riazi G, Pooyan S, Balalaie S. Ac-SDKP ameliorates the progression of experimental autoimmune encephalomyelitis via inhibition of ER stress and oxidative stress in the hippocampus of C57BL/6 mice. Brain Res Bull. 2020;154:21-31. 
9. Chen W, Zhao M, Zhao S, Lu Q, Ni L, Zou C, et al. Activation of the TXNIP/NLRP3 inflammasome pathway contributes to inflammation in diabetic retinopathy: a novel inhibitory effect of minocycline. Inflamm Res. 2017;66:157-66.

10. Hui ST, Andres AM, Miller AK, Spann NJ, Potter DW, Post NM, et al. Txnip balances metabolic and growth signaling via PTEN disulfide reduction. Proc Natl Acad Sci U S A. 2008;105:3921-6.

11. Hou Y, Wang Y, He Q, Li L, Xie H, Zhao Y, et al. Nrf2 inhibits NLRP3 inflammasome activation through regulating Trx1/TXNIP complex in cerebral ischemia reperfusion injury. Behav Brain Res. 2018;336:32-9.

12. Wree A, Eguchi A, McGeough MD, Pena CA, Johnson CD, Canbay A, et al. NLRP3 inflammasome activation results in hepatocyte pyroptosis, liver inflammation, and fibrosis in mice. Hepatology. 2014;59:898-910.

13. Chen $X, H e$ WT, Hu L, Li J, Fang Y, Wang X, et al. Pyroptosis is driven by non-selective gasdermin-D pore and its morphology is different from MLKL channel-mediated necroptosis. Cell Res. 2016;26:1007-20.

14. Somacal S, Figueiredo CG, Quatrin A, Ruviaro AR, Conte L, Augusti PR, et al. The antiatherogenic effect of bixin in hypercholesterolemic rabbits is associated to the improvement of lipid profile and to its antioxidant and anti-inflammatory effects. Mol Cell Biochem. 2015;403:243-53.

15. Tao S, Rojo de la Vega M, Quijada H, Wondrak GT, Wang T, Garcia JG, et al. Bixin protects mice against ventilation-induced lung injury in an NRF2-dependent manner. Sci Rep. 2016;6:18760.

16. Tao S, Lakey-Beitia J, Kumar DJ, Hegde ML, Rao KS. Carotenoids as novel therapeutic molecules against neurodegenerative disorders: Chemistry and molecular docking analysis. Int J Mol Sci. 2019;20:e5553.

17. de Oliveira Júnior RG, Bonnet A, Braconnier E, Groult H, Prunier G, Beaugeard L, et al. Bixin, an apocarotenoid isolated from Bixa orellana L., sensitizes human melanoma cells to dacarbazineinduced apoptosis through ROS-mediated cytotoxicity. Food Chem Toxicol. 2019;125:549-61.

18. Rivera-Madrid R, Aguilar-Espinosa M, Cárdenas-Conejo Y, Garza-Caligaris LE. Carotenoid derivates in achiote (Bixa orellana) seeds: Synthesis and health promoting properties. Front Plant Sci. 2016;7:1406.

19. Xu Z, Kong XQ. Bixin ameliorates high fat diet-induced cardiac injury in mice through inflammation and oxidative stress suppression. Biomed Pharmacother. 2017;89:991-1004.

20. Zhang H, Xue L, Li B, Tian H, Zhang Z, Tao S. Therapeutic potential of bixin in PM2.5 particlesinduced lung injury in an Nrf2-dependent manner. Free Rad Biol Med. 2018;126:166-76.

21. Lu K, Liu L, Xu X, Zhao F, Deng J, Tang X, et al. ADAMTS13 ameliorates inflammatory responses in experimental autoimmune encephalomyelitis. J Neuroinflamm. 2020;17:67.

22. Liu X, Zhu Q, Zhang M, Yin T, Xu R, Xiao W, et al. Isoliquiritigenin ameliorates acute pancreatitis in mice via inhibition of oxidative stress and modulation of the Nrf2/HO-1 pathway. Oxid Med Cell Longev. 2018;2018:7161592. 
23. Qiu X, Guo Q, Liu X, Luo H, Fan D, Deng Y, et al. Pien Tze Huang alleviates relapsing-remitting experimental autoimmune encephalomyelitis mice by regulating Th1 and Th17 cells. Front Pharmacol. 2018;9:1237.

24. Scheiblich $H$, Trombly M, Ramirez A, Heneka MT. Neuroimmune connections in aging and neurodegenerative diseases. Trends Immunol. 2020;41:300-12.

25. Tarantini S, Valcarcel-Ares MN, Yabluchanskiy A, Tucsek Z, Hertelendy P, Kiss T, et al. Nrf2 deficiency exacerbates obesity-induced oxidative stress, neurovascular dysfunction, blood-brain barrier disruption, neuroinflammation, amyloidogenic gene expression, and cognitive decline in mice, mimicking the aging phenotype. J Gerontol A Biol Sci Med Sci. 2018;73:853-63.

26. Milo R, Karussis D. [MULTIPLE SCLEROSIS THERAPY - 2019]. Harefuah. 2019;158:388-94.

27. Dang C, Han B, Li Q, Han R, Hao J. Up-regulation of PGC-1alpha in neurons protects against experimental autoimmune encephalomyelitis. FASEB J. 2019;33:14811-24.

28. Kumar Y, Phaniendra A, Periyasamy L. Bixin triggers apoptosis of human Hep3B hepatocellular carcinoma cells: An insight to molecular and IN SILICO approach. Nutr Can. 2018;70:971-83.

29. Villacampa N, Heneka MT. Microglia in Alzheimer's disease. Local heroes! J Exp Med. 2020;217:e20192311.

30. Khan N, Kuo A, Brockman DA, Cooper MA, Smith MT. Pharmacological inhibition of the NLRP3 inflammasome as a potential target for multiple sclerosis induced central neuropathic pain. Inflammopharmacology. 2018;26:77-86.

31. Zsila F, Molnár P, Deli J, Lockwood SF. Circular dichroism and absorption spectroscopic data reveal binding of the natural cis-carotenoid bixin to human alpha1-acid glycoprotein. Bioorg Chem. 2005;33:298-309.

32. Guo C, Fulp JW, Jiang Y, Li X, Chojnacki JE, Wu J, et al. Development and characterization of a hydroxyl-sulfonamide analogue, 5-chloro-N-[2-(4-hydroxysulfamoyl-phenyl)-ethyl]-2-methoxybenzamide, as a novel NLRP3 inflammasome inhibitor for potential treatment of multiple sclerosis. ACS Chem Neurosci. 2017;8:2194-201.

33. Anwar S, Rivest S. Alzheimer's disease: microglia targets and their modulation to promote amyloid phagocytosis and mitigate neuroinflammation. Expert Opin Ther Targets. 2020;24:331-44.

34. Braga TT, Brandao WN, Azevedo H, Terra FF, Melo ACL, Pereira FV, et al. NLRP3 gain-of-function in CD4 + T lymphocytes ameliorates experimental autoimmune encephalomyelitis. Clin Sci (Lond). 2019;133:1901-16.

35. Soares JL, Oliveira EM, Pontillo A. Variants in NLRP3 and NLRC4 inflammasome associate with susceptibility and severity of multiple sclerosis. Mult Scler Relat Disord. 2019;29:26-34.

36. Lu L, Lu Q, Chen W, Li J, Li C, Zheng Z. Vitamin D3 protects against diabetic retinopathy by inhibiting high-glucose-induced activation of the ROS/TXNIP/NLRP3 inflammasome pathway. J Diabetes Res. 2018;2018:8193523.

37. Han Y, Xu X, Tang C, Gao P, Chen X, Xiong X, et al. Reactive oxygen species promote tubular injury in diabetic nephropathy: The role of the mitochondrial ros-txnip-nlrp3 biological axis. Redox Biol. 
2018;16:32-46.

38. Wang DS, Yan LY, Yang DZ, Lyu Y, Fang LH, Wang SB, et al. Formononetin ameliorates myocardial ischemia/reperfusion injury in rats by suppressing the ROS-TXNIP-NLRP3 pathway. Biochem Biophys Res Commun. 2020;525:759-66.

39. Lian D, Dai L, Xie Z, Zhou X, Liu X, Zhang Y, et al. Periodontal ligament fibroblasts migration injury via ROS/TXNIP/NIrp3 inflammasome pathway with Porphyromonas gingivalis lipopolysaccharide. Mol Immunol. 2018;103:209-19.

40. Tan Y, Ichikawa T, Li J, Si Q, Yang H, Chen X, et al. Diabetic downregulation of Nrf2 activity via ERK contributes to oxidative stress-induced insulin resistance in cardiac cells in vitro and in vivo.

Diabetes. 2011;60:625-33.

41. Murakami S, Motohashi H. Roles of Nrf2 in cell proliferation and differentiation. Free Rad Biol Med. 2015;88:168-78.

42. Rojo de la Vega M, Zhang DD, Wondrak GT. Topical bixin confers NRF2-dependent protection against photodamage and hair graying in mouse skin. Front Pharmacol. 2018;9:287.

43. Xue L, Zhang H, Zhang J, Li B, Zhang Z, Tao S. Bixin protects against particle-induced long-term lung injury in an NRF2-dependent manner. Toxicol Res (Camb). 2018;7:258-70.

\section{Figures}


a

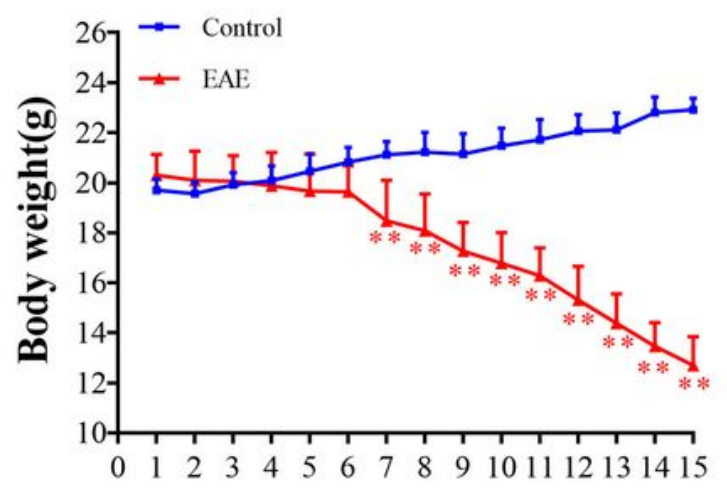

Times(Days)

c

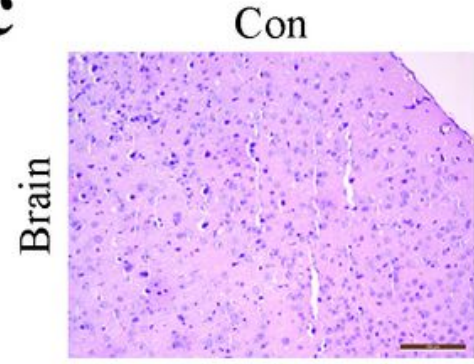

e<smiles>COC(=O)/C=C/C(C)=C/C=C/C(C)=C/C=C/C=C(C)/C=C/C=C(C)/C=C/C(=O)OC</smiles>

Bixin

g
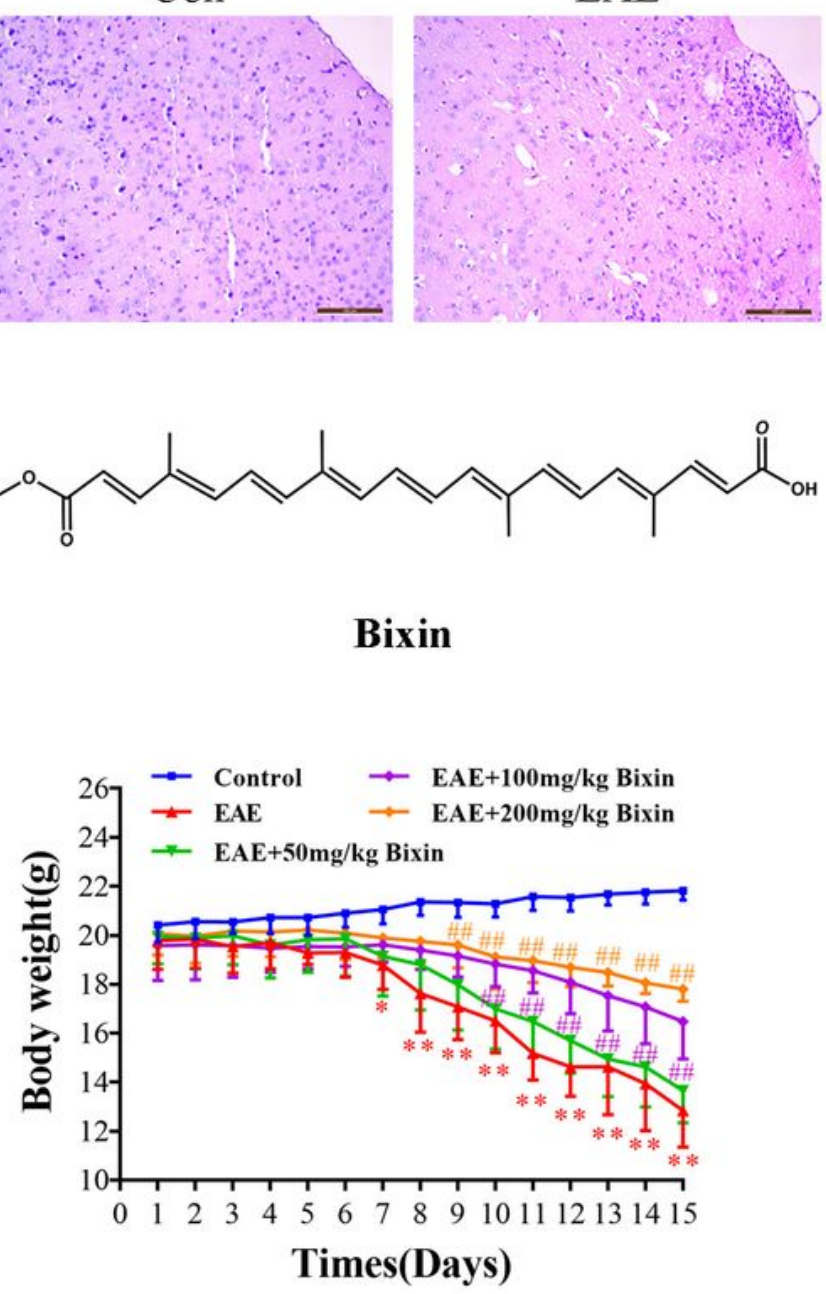

b

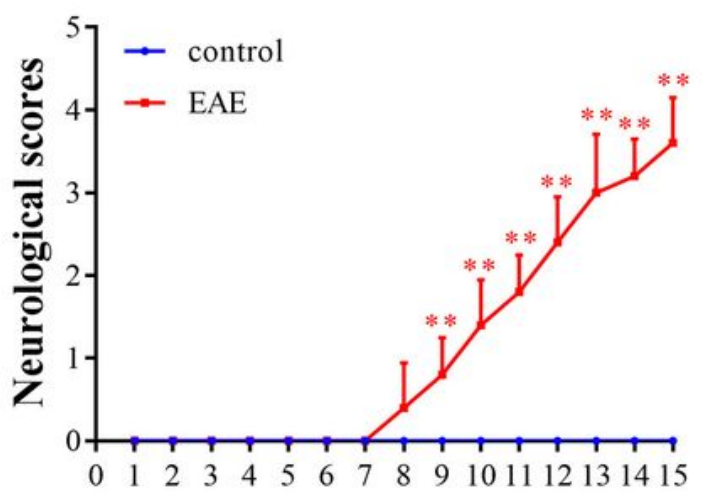

Times(Days)

d

Con

EAE
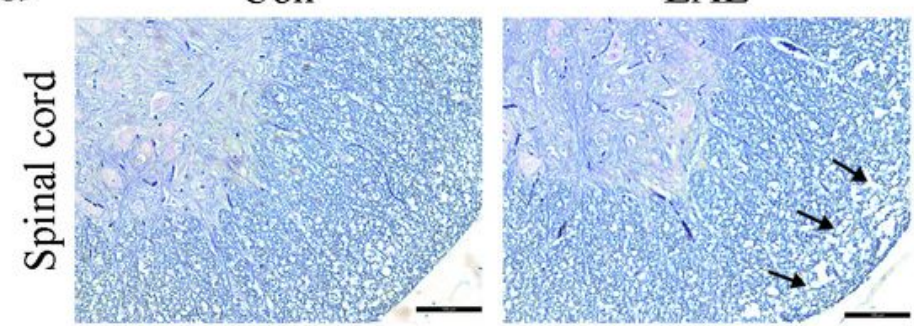

$f$

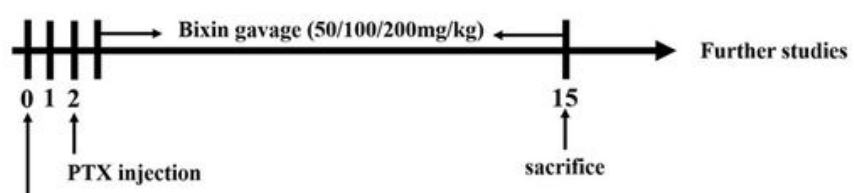

$\mathrm{h}$

MOG injection

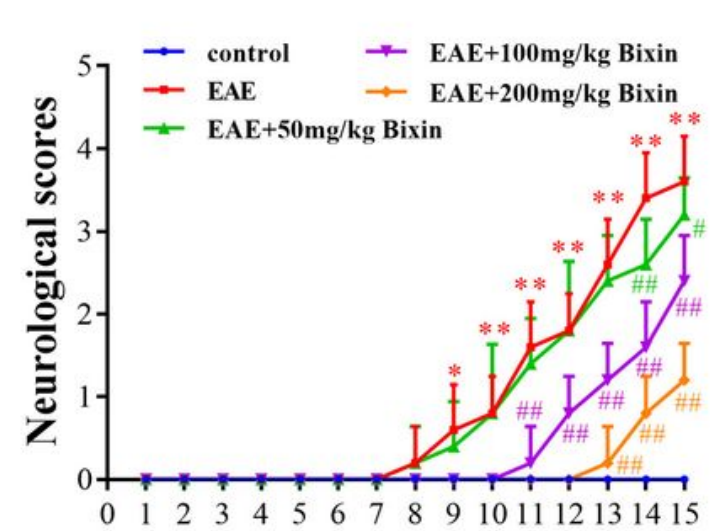

Times(Days)

\section{Figure 1}

Bixin attenuates the symptoms of EAE mice. $a$, b bodyweights and clinical behavior scores of the EAE mice. c HE staining showing inflammatory cell infiltration in the brain. Scale bars: $100 \mu \mathrm{m}$. d LFB staining showing demyelination in the spinal cord. Scale bars: $100 \mu \mathrm{m}$. e The chemical structure of bixin. $f$ The procedure of this study. $\mathrm{g}, \mathrm{h}$ Changes in bodyweights and clinical behavior scores of EAE mice during the 
experimental period. Data shown in graphs represents the means $\pm S D(n=5) .{ }^{*} P<0.05,{ }^{*} P<0.01$, vs. control group; \# $\mathrm{P}<0.05$, \#\# $\mathrm{P}<0.01$, vs. EAE group.

$\mathrm{a}$

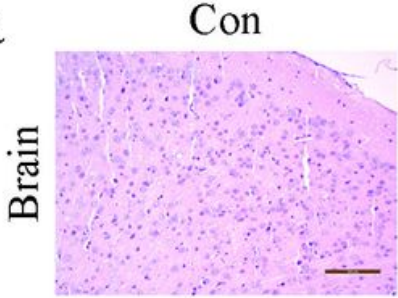

b
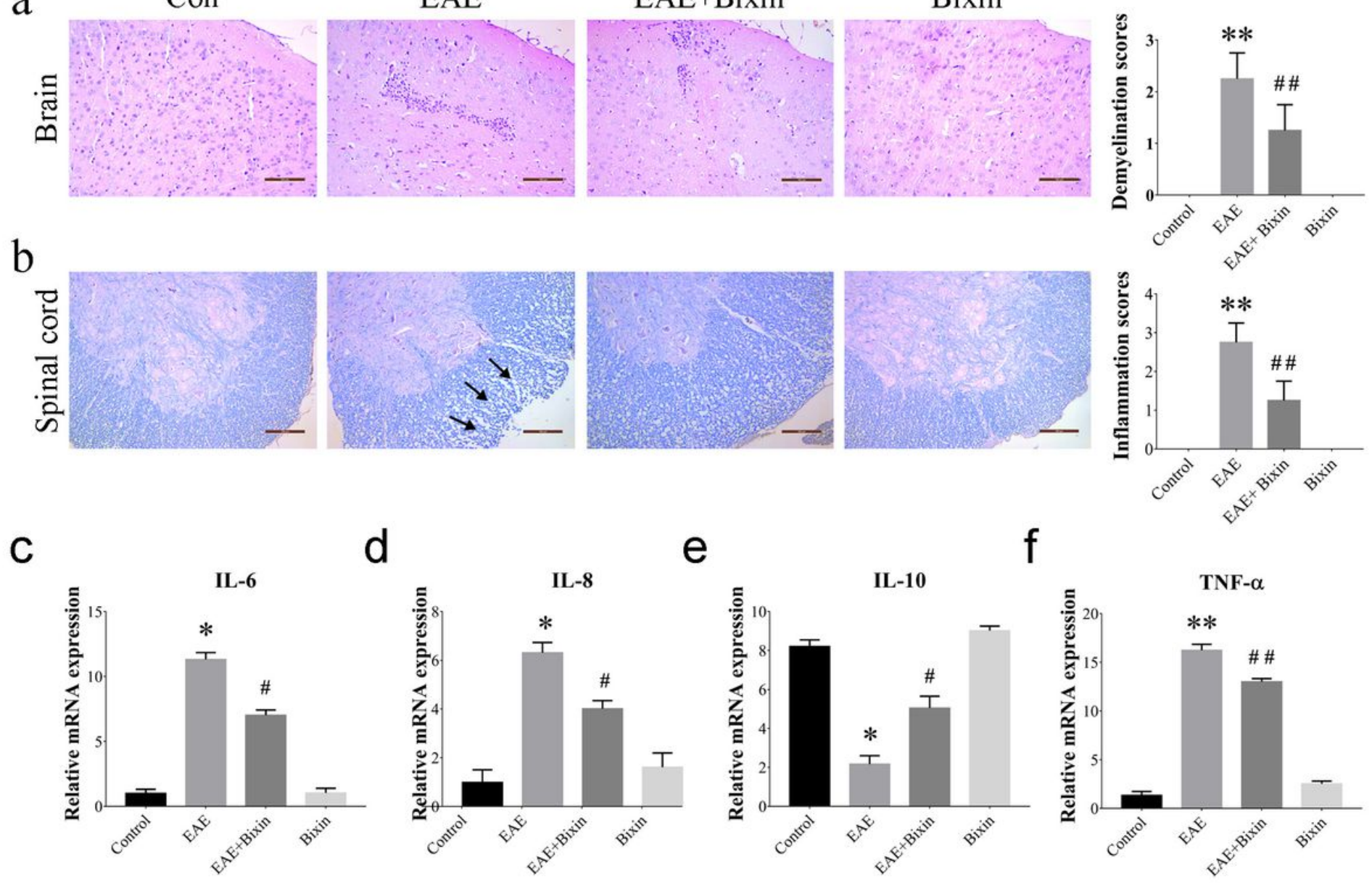

$\mathrm{g}$

Con

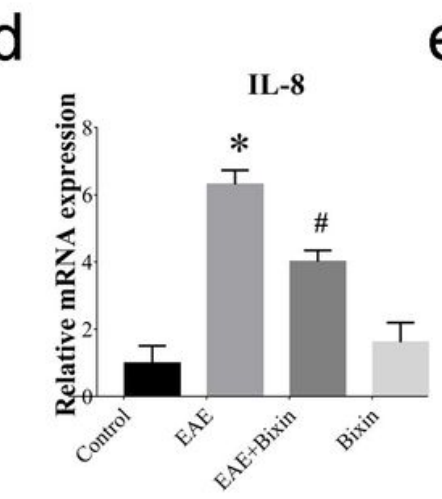

EAE e

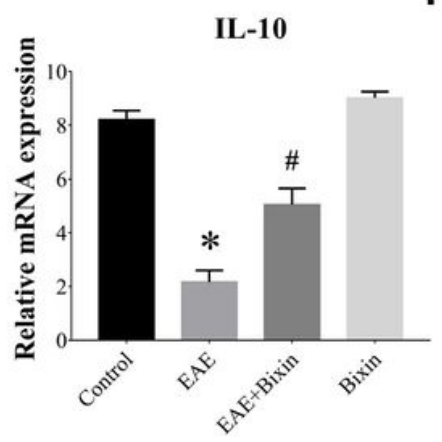

EAE+Bixin
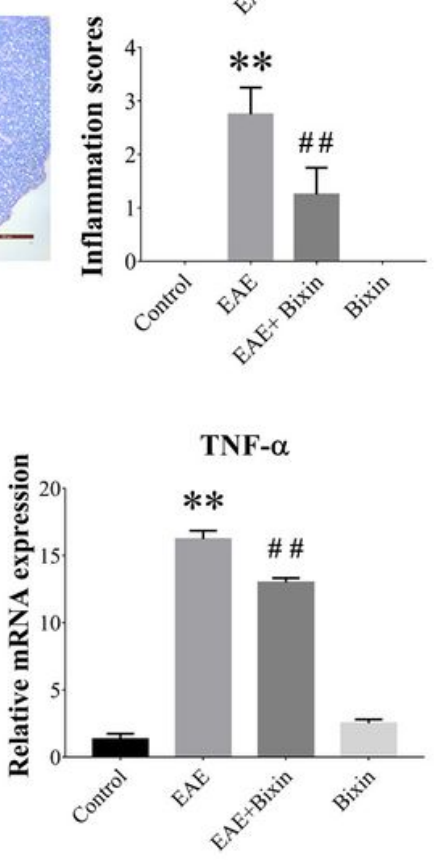

Bixin
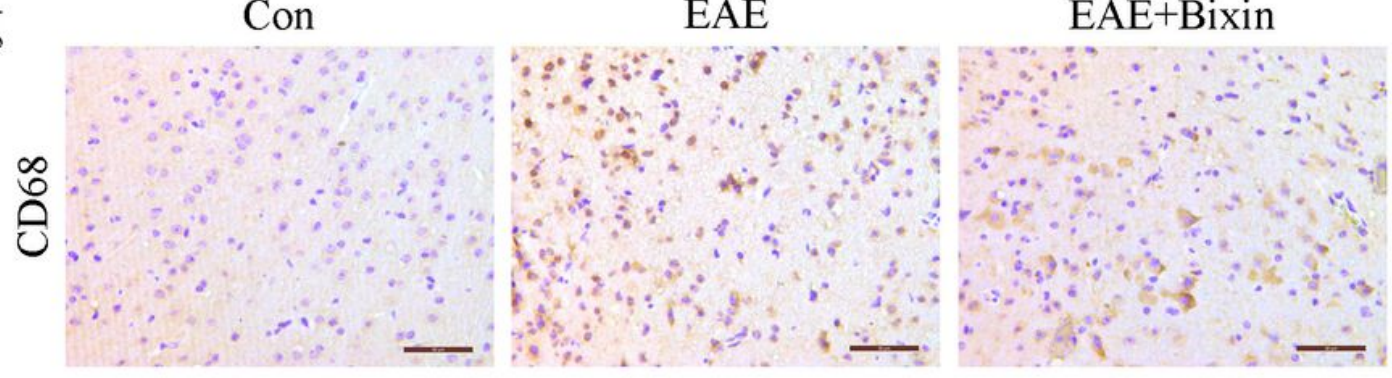

\section{Figure 2}

Bixin reduces the inflammation and demyelination in EAE mice. a HE staining showing inflammatory cell infiltration in the brain, as well as the inflammation scores. Scale bars: $100 \mu \mathrm{m}$. b LFB staining showing demyelination in the spinal cord, as well as the demyelination scores. Scale bars: $100 \mu \mathrm{m}$. c-f mRNA expression of the inflammatory cytokines TNF-a, IL-6, IL-8 and IL-10 were quantitated by qRT-PCR. $\mathrm{g}$ Representative IHC images of CD68 in brain sections showing the activated microglia. Scale bars: $50 \mu \mathrm{m}$. Data shown in graphs represents the means $\pm S D(n=5)$. ${ }^{*}<<0.05$, $* * P<0.01$, vs. control group; \#P< 0.05 , \#\# $P<0.01$, vs. EAE group. 

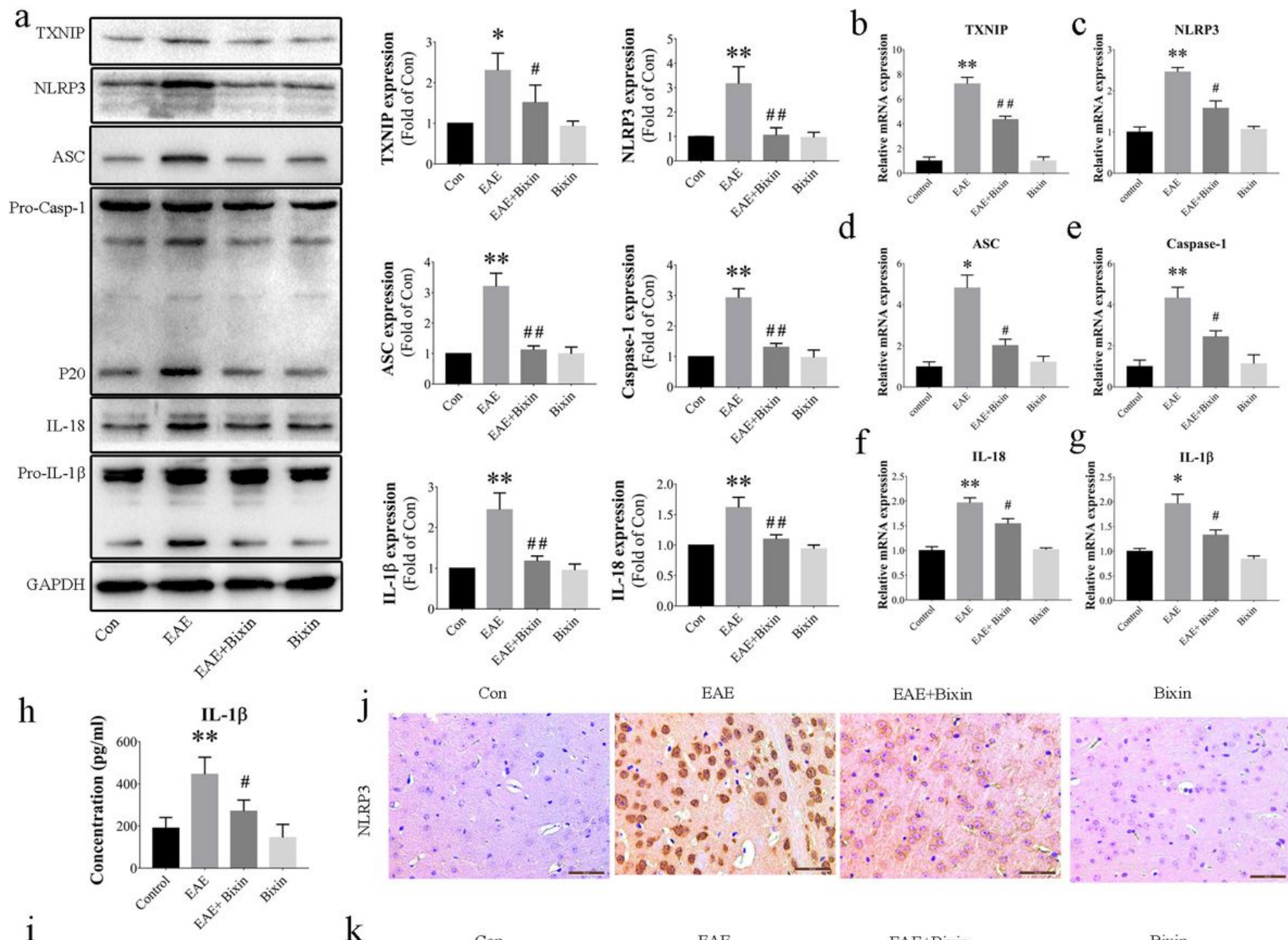

i

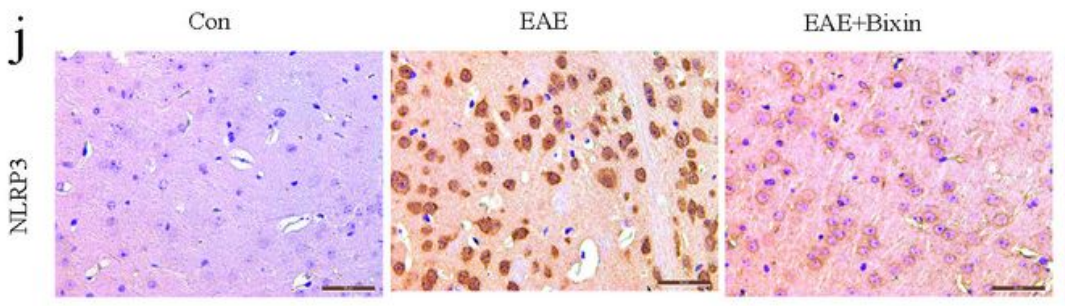

$\mathrm{k}$
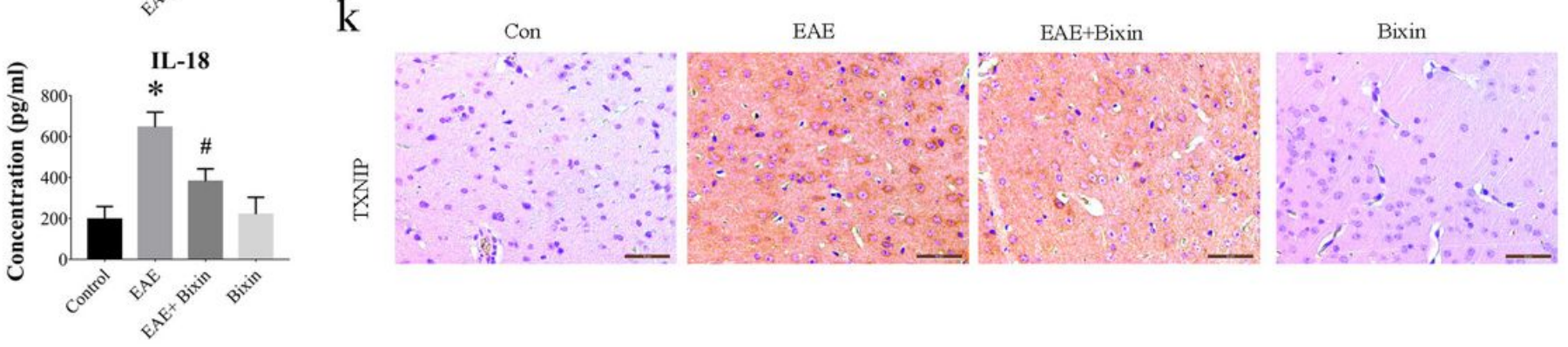

\section{Figure 3}

Bixin suppresses the activation of the TXNIP/NLRP3 inflammasome in EAE mice. a The protein levels of TXNIP/NLRP3 inflammasome components were assessed by western blotting, and the quantitative results are shown. b-g mRNA expression of TXNIP/NLRP3 inflammasome components were quantitated by qRT-PCR. $h$-i Brain tissues IL-1 $\beta$ and IL-18 levels were measured by ELISA. j-k Representative IHC images of NLRP3 and TXNIP in brain sections. Scale bars: $50 \mu \mathrm{m}$. Data shown in graphs represents the means $\pm S D(n=5)$. ${ }^{P}<0.05,{ }^{*} P<0.01$, vs. control group; \# $P<0.05$, \#\# $P<0.01$, vs. EAE group. 
a Con EAE EAE+Bixin
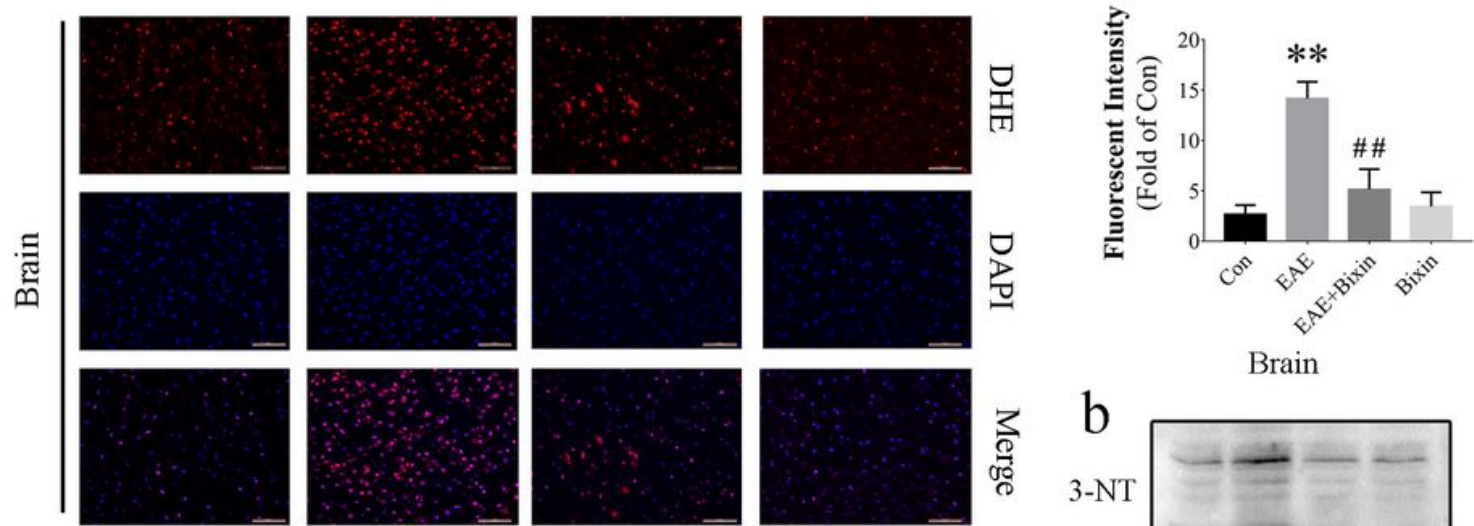

Brain
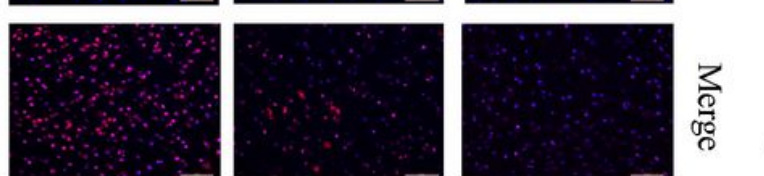

b
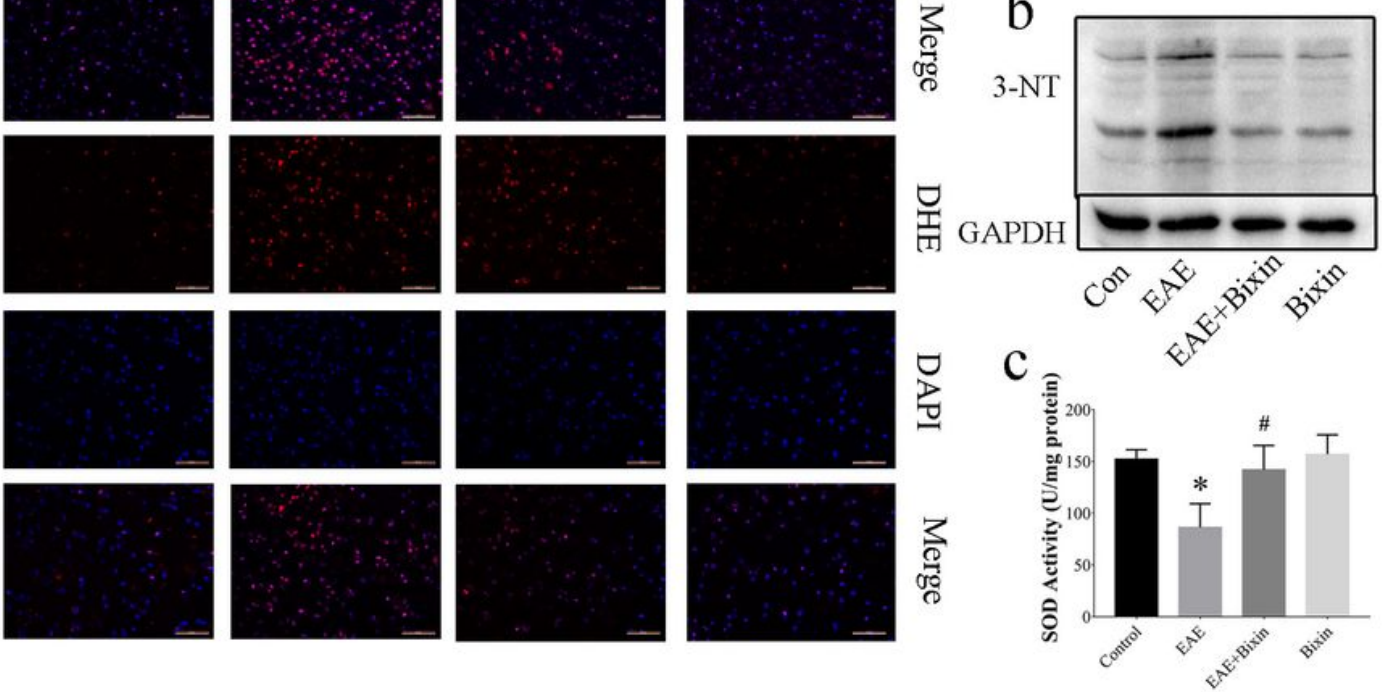

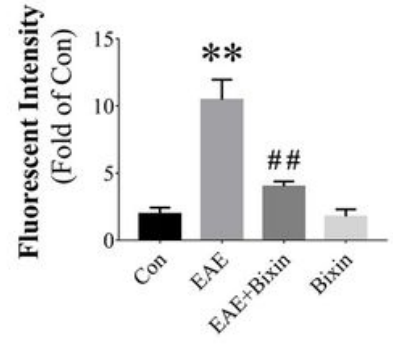

Spinal cord

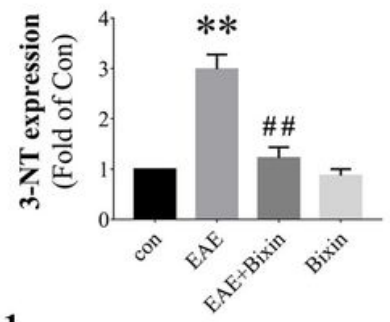

d

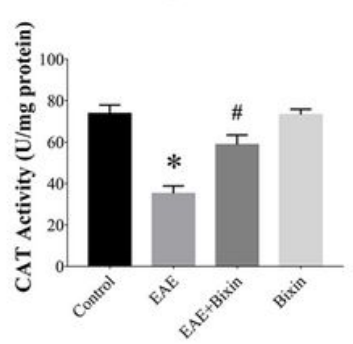

\section{Figure 4}

Bixin alleviates oxidative stress in EAE mice. a The level of reactive oxygen species (ROS) in the brain and spinal cord tissues was assessed by DHE staining, and the quantitative results are shown. Scale bars: 50 $\mu \mathrm{m}$. b The protein level of 3-NT in the brain tissues was assessed by western blotting, and the quantitative results are shown. c-d The activities of SOD and CAT in the brain tissues. Data shown in graphs represents the means $\pm S D(n=5)$. ${ }^{*}<<0.05$, ${ }^{\star} P<0.01$, vs. control group; \# $P<0.05$, \#\# $P<0.01$, vs. EAE group. 
a

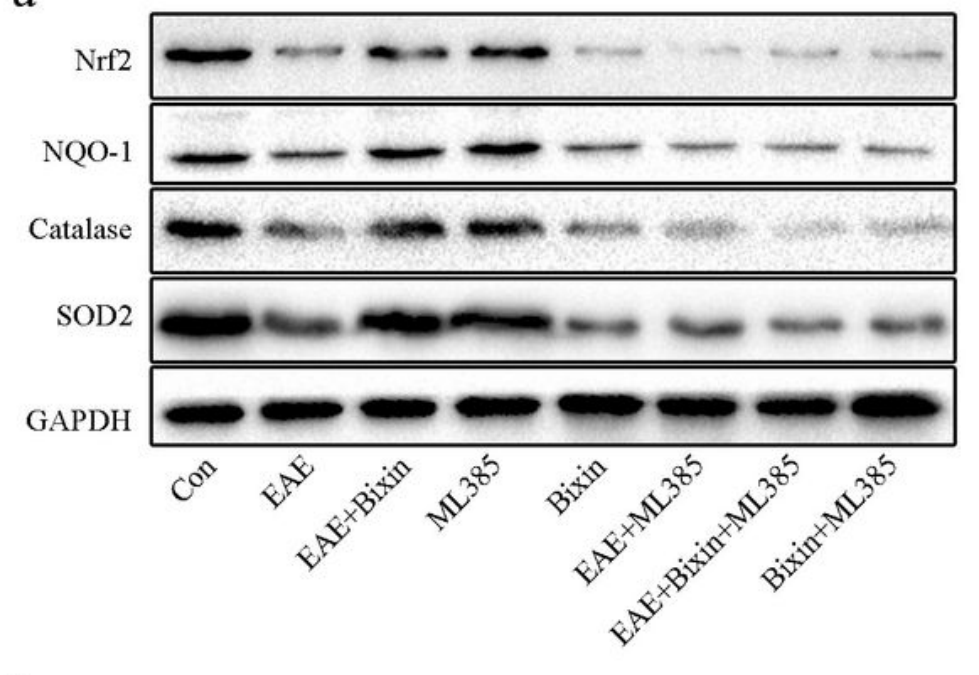

b

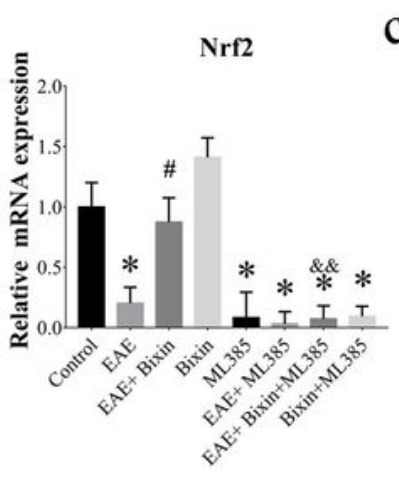

f

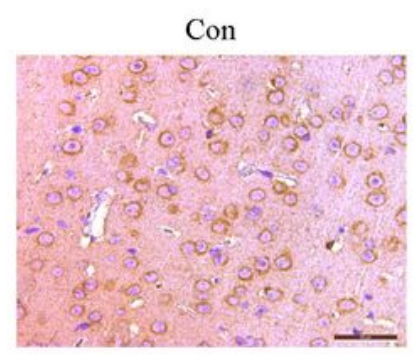

ML385

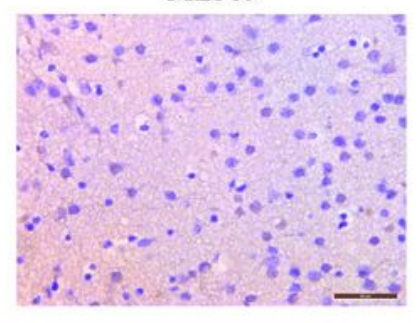

d

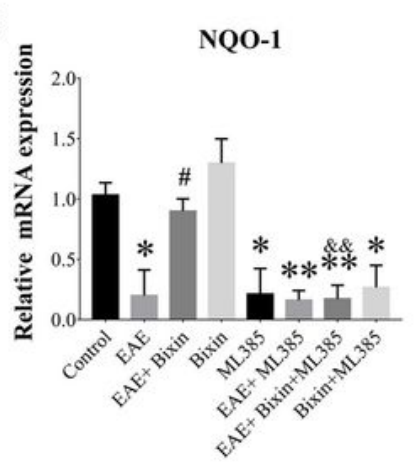

EAE

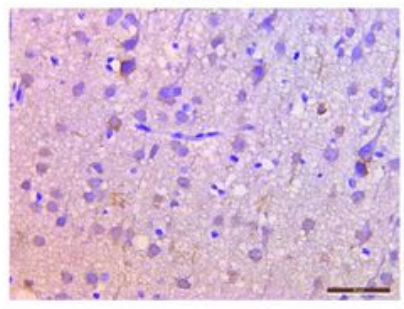

EAE+ML385

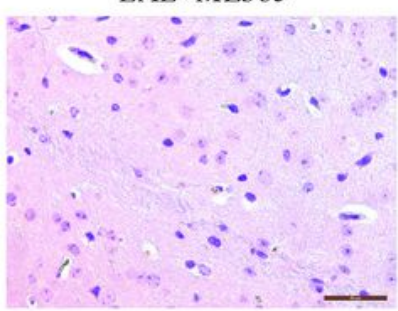

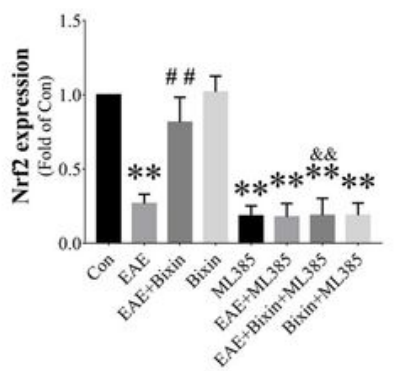
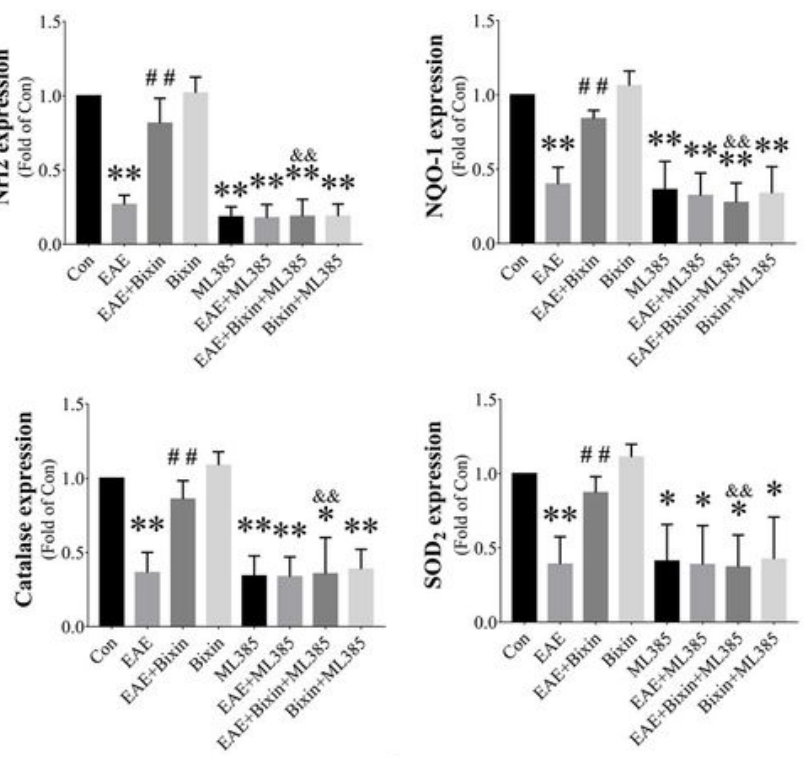

e
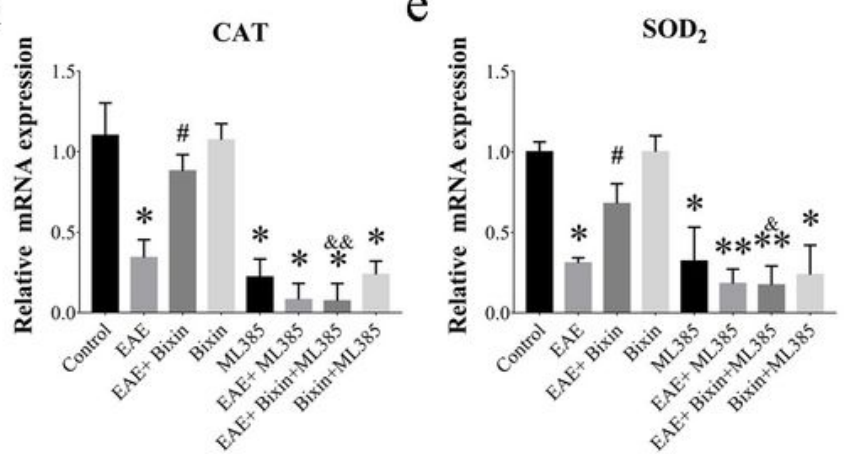

$\mathrm{EAE}+\mathrm{Bixin}$

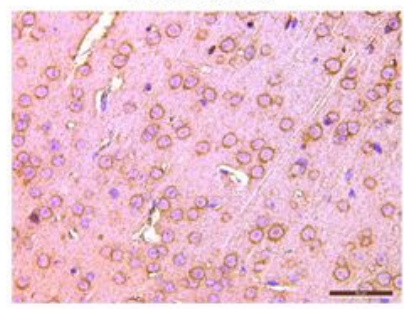

EAE+ML385+Bixin

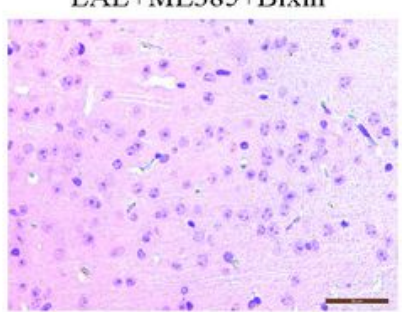

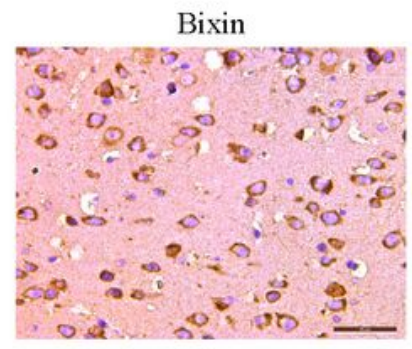

Bixin+ML385

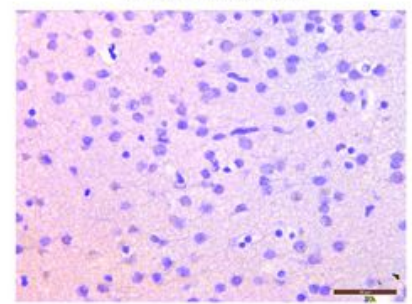

\section{Figure 5}

Bixin activates the Nrf2 signaling in EAE mice. a The protein levels of Nrf2 and downstream genes Catalase, NQO-1 and SOD2 in the brain tissues were assessed by western blotting, and the quantitative results are shown. b-e mRNA expression of Nrf2, Catalase, NQO-1 and SOD2 in the brain tissues were quantitated by qRT-PCR. $f$ IHC of Nrf2 in brain sections. 

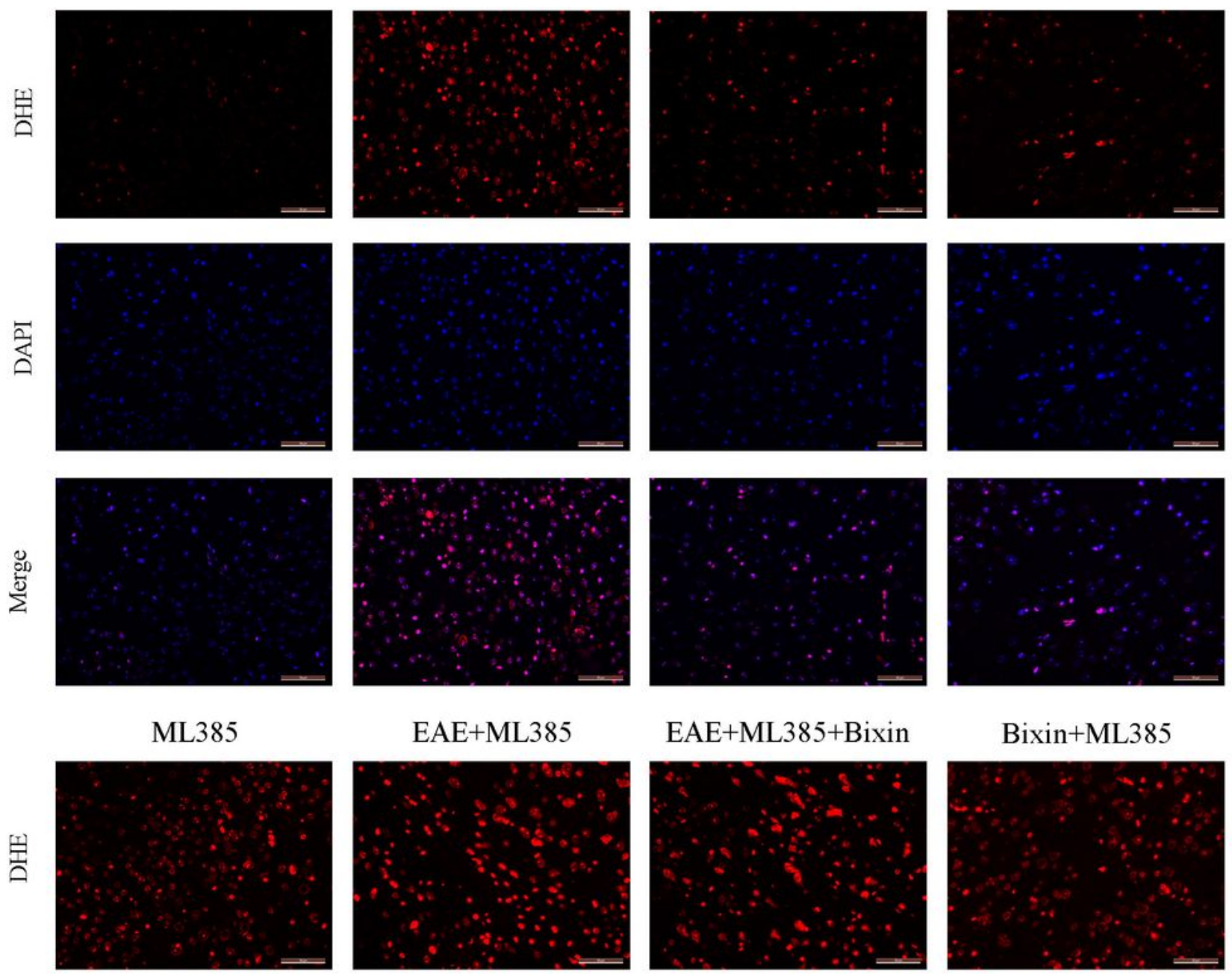

$\mathrm{EAE}+\mathrm{ML} 385+\mathrm{Bixin}$

\section{Bixin+ML385}
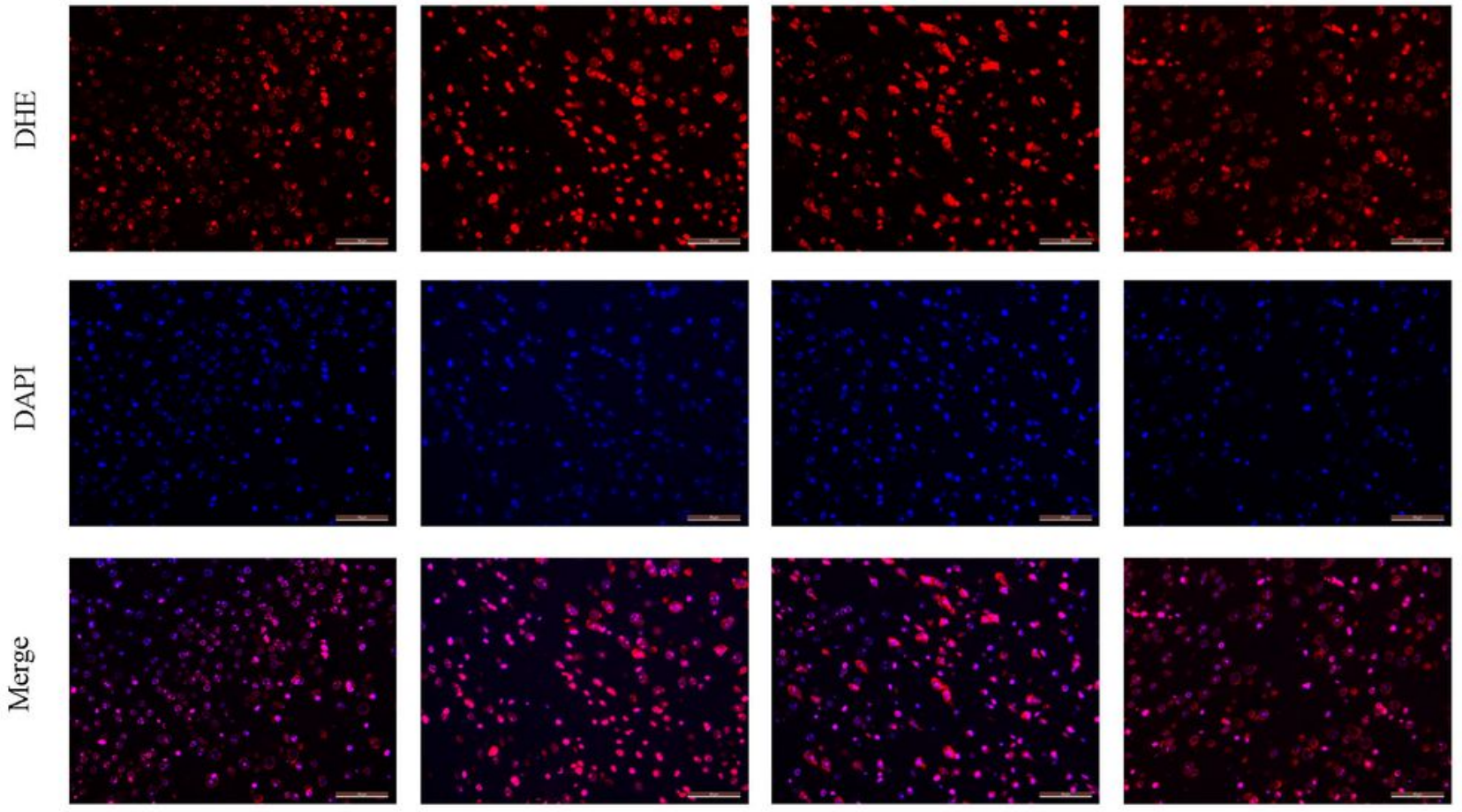

\section{Figure 6}

Bixin activates the Nrf2 signaling in EAE mice. $g$ The level of reactive oxygen species (ROS) in the brain tissues was assessed by DHE staining. Scale bars: $50 \mu \mathrm{m}$. Data shown in graphs represents the means \pm $S D(n=5)$. ${ }^{*}<<0.05, * * P<0.01$, vs. control group; \#P<0.05, \#\#P<0.01, vs. EAE group; \&P< 0.05, \&\&P< 0.01 , vs. EAE + bixin group. 